\title{
Contribution to Development of Reliability and Optimization Methods Applied to Mechanical Structures
}

\author{
Siham Ouhimmou ${ }^{1}$, Abdelkhalak El Hami ${ }^{2}$, Rachid Ellaia ${ }^{3}$, Mohamed Tkiouat ${ }^{3}$ \\ ${ }^{1}$ Laboratory of Mechanics, University Hassan II, Casablanca, Morocco \\ ${ }^{2}$ Laboratory of Mechanics of Rouen, National Institute of Applied Sciences, Rouen, France \\ ${ }^{3}$ Laboratory of Study and Research in Mathematics Applied, Mohammedia School of Engineers, \\ Agdal-Rabat, Morocco \\ Email: ouhimmousiham@yahoo.fr, siham25@gmail.com
}

Received June 11, 2012; revised November 8, 2012; accepted November 15, 2012

\begin{abstract}
In order to take into account the uncertainties linked to the variables in the evaluation of the statistical properties of structural response, a reliability approach with probabilistic aspect was considered. This is called the Probabilistic Transformation Method (PTM). This method is readily applicable when the function between the input and the output of the system is explicit. However, the situation is much more involved when it is necessary to perform the evaluation of implicit function between the input and the output of the system through numerical models. In this work, we propose a technique that combines Finite Element Analysis (FEA) and Probabilistic Transformation Method (PTM) to evaluate the Probability Density Function (PDF) of response where the function between the input and the output of the system is implicit. This technique is based on the numerical simulations of the Finite Element Analysis (FEA) and the Probabilistic Transformation Method (PTM) using an interface between Finite Element software and Matlab. Some problems of structures are treated in order to prove the applicability of the proposed technique. Moreover, the obtained results are compared to those obtained by the reference method of Monte Carlo. A second aim of this work is to develop an algorithm of global optimization using the local method SQP, because of its effectiveness and its rapidity of convergence. For this reason, we have combined the method SQP with the Multi start method. This developed algorithm is tested on test functions comparing with other methods such as the method of Particle Swarm Optimization (PSO). In order to test the applicability of the proposed approach, a structure is optimized under reliability constraints.
\end{abstract}

Keywords: Reliability Methods; Probabilistic Transformation Method; Finite Element Analysis; FEACPTM; The Method SQP; The Multi Start Method; Algorithm MSQP; Structural Optimization

\section{Introduction}

As the properties of the realistic structure, the model is necessary to take into account some uncertainty. This uncertainty can be conveniently described in terms of probability measures, such as distribution functions. It is a major goal of reliability methods to relate the uncertainties of the input variables to the uncertainty of the structural performance. A fundamental problem in structural reliability analysis is the computation of the probability integral ([1-5]):

$$
P_{f}=\operatorname{Prob}[G(X) \leq(0)]=\int_{G(X) \leq 0} f(X) \mathrm{d} X
$$

where $X=\left[X_{1}, \cdots, X_{n}\right]^{\mathrm{T}}$ in which $\mathrm{T}$ is the transpose, is a vector of random variables representing the uncertain parameters of considered structure, $f(X)$ is the prob- ability density function of $X, G(X)$ is the Limit State Function defined such that: $G(X) \leq 0$ is the domain of integration denoted the failure set, and $P_{f}$ is the probability of failure. The difficulty of computing this integration led to development of various methods of reliability analysis such as Monte Carlo, FORM and SORM ([6-8]), and Probabilistic Transformation Method PTM.

In this paper, a proposed Method: Finite Element Analysis (FEA) coupled with the Probabilistic Transformation Method (PTM) is applied in order to evaluate numerically the probabilistic and statistical characteristics of the response of stochastic mechanical system. It involves four main steps: 1) sampling on input random variables; 2) using Finite Element Analysis (FEA) software to have the response variable of system; 3) estimating the Probabilistic Density Function (PDF) of this response variable using the Probabilistic Transformation 
Method PTM program; and 4) concluding the probability of failure and reliability of systems. To show the advantage of the proposed method, we have carried out different applications to cover several structural problems.

\section{Reliability Method of Analysis}

\subsection{Finite Element Analysis}

The finite element method is the standard tool for certain classes of partial differential equations arising in various fields of engineering and in particular for those arising in solid mechanics. For linear systems enforcing global static or dynamic equilibrium, the FE method leads to a system of linear equations, respectively

$$
\begin{gathered}
K U=F \\
M \ddot{U}(t)+C \dot{U}(t)+K U(t)=F(t)
\end{gathered}
$$

where the matrix $K$ is the global stiffness matrix, $M$ is the mass matrix, $U$ the vector of displacement, $F$ the vector of applied loads, $C$ is the damping matrix.

These matrix obtained by adding the contributions of all element matrix. There are symmetrical and positive vectors.

$$
\begin{aligned}
K & =\sum_{e} K^{e} \\
M & =\sum_{e} M^{e}
\end{aligned}
$$

The latter matrix has the form,

$$
\begin{gathered}
K^{e}=\int_{\Omega^{e}} D^{e} B^{e} \mathrm{~d} \Omega^{e} \\
M^{e}=\int_{\Omega^{e}} \rho^{e} H^{e T} H^{e} \mathrm{~d} \Omega^{e}
\end{gathered}
$$

where $B^{e}$ is the matrix relating element displacements and strains, $D^{e}$ is the elasticity matrix relating stresses and strains, $\rho^{e}$ is the mass density, $H^{e}$ is the shape functions and $\Omega^{e}$ is the spatial domain of the element. The global damping matrix $C$ is typically formulated in terms of $M$ and $K$.

\subsection{Probabilistic Transformation Method PTM}

The Probabilistic Transformation Method is based on the following theorem:

Theorem: Suppose that $X$ is a continuous random variable with PDF $f(x)$ and $A \subset \Re$ is the one-dimensional space where $f(x) \succ 0$, is differentiable and monotonic. Consider the random variable $Y=u(X)$, where $y=u(x)$ defines a one-to-one transformation that maps the set $A$ onto a set $B \subset \mathfrak{R}$ so that the equation $y=u(x)$ can be uniquely solved for $x$ in terms of $y$, say $x=u^{-1}(y)$. Then, the PDF of $Y$ is

$$
f_{Y}(Y)=f_{X}\left[u^{-1}(y)\right]|J|
$$

where, $J=\frac{\mathrm{d} x}{\mathrm{~d} y}=\frac{\mathrm{d} u^{-1}(y)}{\mathrm{d} y}$ is the Jacobean transformation, which must be continuous for all points $y \in B$.

The PTM is based on one-to-one mapping between the random output(s) and input(s) where the transformation $J$. Jacobean can be computed. The PDF of the output(s) is then computed through the known joint PDF of the inputs multiplied by the determinant of the Jacobean matrix.

The idea of PTM is based on the following formula [9]:

$$
\begin{aligned}
& f_{u}(u)=|J| \cdot f_{z}(z) \\
& |J|=\left|\frac{\partial z}{\partial u}\right|
\end{aligned}
$$

$f_{u}(u)$ is the probability density function of the variable $u$.

$f_{z}(z)$ is the probability density function of the variable $z$.

The Probabilistic Transformation Method (PTM) is one of the most widely used methods in reliability analysis. However, this method has drawbacks in the solution of reliability problems. It requires the evaluation of the explicit response functions with respect to the random variables that is very difficult in analysis of complicated structures. To overcome these drawbacks, an interface between the Finite Element Analysis and The Probabilistic Transformation Method (PTM) is proposed in this paper.

\subsection{Finite Element Analysis Coupled with Probabilistic Transformation Method: FEACPTM}

The Finite Element Analysis (FEA) software is used to perform the structural analysis to obtain the maximal displacement of the structure, and maximal stress, corrsponding to a set of given design variables ([10-12]). These analysis results are sent to the reliability program to conduct the Probabilistic Density Function PDF, and the probability of failure and generate new random variables. The newly generated variables are then used to update the input file. The Finite Element Analysis (FEA) software is then invoked again to perform the structural analysis with the new input parameters. This process is repeated until satisfactory results are obtained.

\subsubsection{Interface between FEA Software and Reliability Analysis Program}

A fundamental characteristic of a software code for reliability analysis of structural engineering applications consists in the way it interfaces with the software that gives the finite element modelling and solution. In this 
type of implementation, the FEA is viewed as a black box in the analysis process and the FE code is communicated with through a generic interface, through the input files of the latter. The reliability analysis program controls the FE code by automatically modifying the input files, set identifiers, which govern the automatic generation of input file samples by the stochastic solver, using pattern matching and replacement.

\subsubsection{Algorithm of Method FEACPTM}

The outline of a Proposed Method FEACPTM is as follows:

1) Generate the input random variables;

2) Calculate the value of output variables by FE software. For each value of input the correspondent value of output is estimating using FEA and stocking it in solution file;

3) Approximate the function between input and output variables using Spline Interpolation;

4) Calculate of the determinant of Jacobean of input and output variables (Equation (9));

5) Apply the basic relation of PTM (Equation (8));

6) Evaluate the graphic of PDF of output variable in function of this output variables (in our case the PDF of displacement in function of displacement);

7) Approximate the Probability of failure $P_{f}$.

\subsection{Application}

We are going to analyze the reliability of the pylon of a line of transportation of electricity that one assimilates to a truss plan. Two identical loads $\mathrm{F}$ of $1.8 \mathrm{KN}$ are applied to the two superior extremities of the following pylon an angle of $\theta=15$. The bars forming the pylon are in steel of which Young's modulus $E=[100 \mathrm{GPa}, 300 \mathrm{GPa}]$ and the Poisson coefficient $\sigma=0.29$. The section of every bar is worth $A=27.90 \mathrm{~cm}^{2}$. The hypothesis for this problem is that the weight of each bar of the pylon is negligible in front of the applied efforts (see Figure 1).

This structure is analyzed using the FEA software for structural modelling, and static analysis in which the FEA software is used to approximate the structural response, this response is used by the PTM program for computing the probability of failure. For that purpose, statistic models must be defined for each random variable involved. In this case, the Young's modulus $E$ is uniformly distributed in the range [100 GPa, $300 \mathrm{GPa}$ ]. Using the proposed technique FEACPTM, we obtain the following graph (see Figure 2):

The PDFs of the normalized vertical displacement $u_{y}$ are plotted in Figure 2 assuming that the variable $u_{y}$ is independent and uniformly distributed in the range [2.9489e04, 8.8466e04]. Also in this case the results are accurate as shown by favourable comparison with classical Monte Carlo simulation. Let us suppose the limit

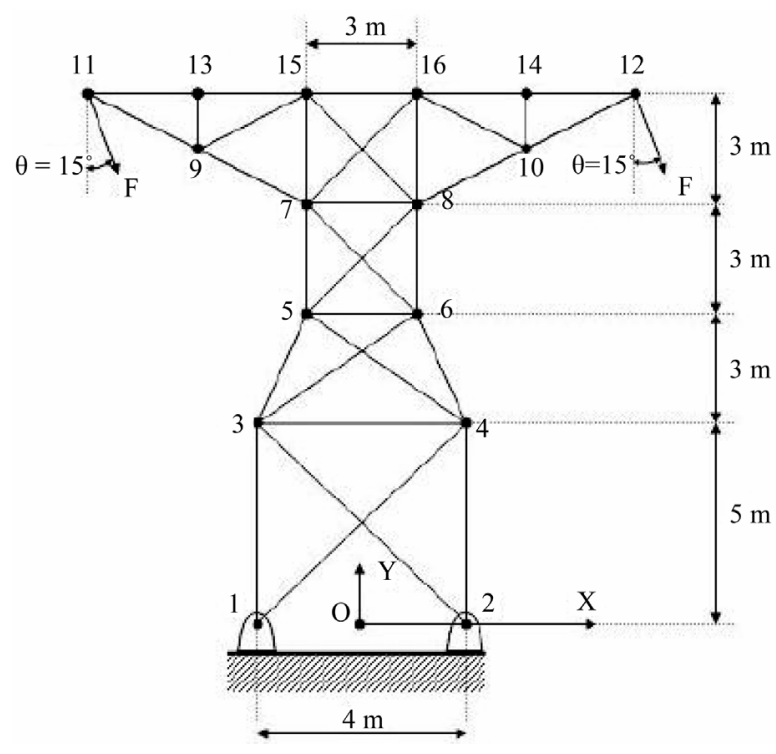

Figure 1. The pylon.

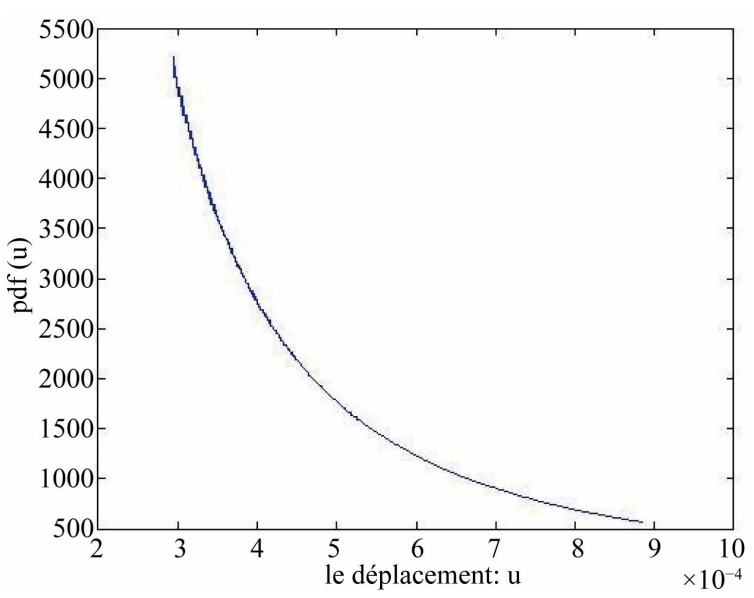

Figure 2. PDF (u) when $E$ is uniformly distributed.

displacement is $u=6.635 \mathrm{e} 04 \mathrm{~mm}$. It is required to find the failure probability $P_{f}=P\left(u \leq u_{\text {limit }}\right)$

Mean Squared Error (MSE) and Absolute Percentage Error (APE) are calculated by using the following equation

$$
\begin{aligned}
& M S E=\left(u_{1}-u_{m c}\right)^{2} \\
& A P E=\left|\frac{\left(u_{1}-u_{m c}\right)}{u_{m c}} \times 100\right|
\end{aligned}
$$

where $u_{1}$ the value of displacement obtained with FEACPTM is, $u_{m c}$ is the predicted value with Monte Carlo simulation.

The numerical values of probabilistic characteristics of the displacement of this pylon are listed in this table.

Table 1 reports the results obtained by our technique and the Monte Carlo simulation (10,000 iterations). This table also illustrates the efficiency of the FEACPTM, 
Table 1. Results obtained by FEACPTM and Monte Carlo simulation.

\begin{tabular}{ccccc}
\hline & FEAPTM & Monte Carlo & MSE & $\begin{array}{c}\text { APE } \\
(\%)\end{array}$ \\
\hline$u_{\min }$ & $2.9489 \mathrm{e}-04$ & $2.9490284 \mathrm{e}-04$ & $1.6487 \mathrm{e}-16$ & 0.0044 \\
$u_{\max }$ & $8.8466 \mathrm{e}-04$ & $8.8460969 \mathrm{e}-04$ & $2.5311 \mathrm{e}-15$ & 0.0057 \\
$u_{\text {mean }}$ & $4.8605 \mathrm{e}-04$ & $4.8594847 \mathrm{e}-04$ & $1.0308 \mathrm{e}-14$ & 0.0209 \\
Var & $1.5756 \mathrm{e}-04$ & $1.5725825 \mathrm{e}-04$ & $9.1053 \mathrm{e}-14$ & 0.1919 \\
$P_{f}$ & $1.8856 \mathrm{e}-01$ & $1.66693 \mathrm{e}-01$ & $4.7817 \mathrm{e}-04$ & 13.1181 \\
\hline
\end{tabular}

since a number of 800 (less than 1000) iterations suffice to obtain results close to those obtained by Monte Carlo simulation. To compare the results, the MSE and APE are calculated. The values of MSE and APE are very small, which shows the accuracy and efficiency of FEACPTM.

\section{Optimization Method of Analysis}

\subsection{Sequential Quadratic Programming (SQP) Method}

We present Sequential Quadratic Programming (SQP) method for optimization problems involving general linear and nonlinear constraints. This method has proved highly effective for many such problems. It typically finds a (local) optimum from an arbitrary starting point, and they require relatively few evaluations of the problem functions and gradients. The method SQP consist in to resolve an optimization problem for a limited number of variables under the following general form ([13,14]):

$$
\left\{\begin{array}{cc}
\text { Minimize } & f(X), X \in \mathfrak{R} \\
\text { Subject to } & h(X)=0 \\
g(X) \leq 0
\end{array}\right.
$$

where $f(X)$ is the objective function, $X$ is the vector of independents variables of optimization and $h(X)$ is the equality constraints, $g(X)$ are called "inequality constraints". An SQP method obtains search directions from a sequence of (Quadratic Problem: QP) sub-problems. Each QP sub-problem minimizes a quadratic model of a certain lagrangian function subject to linearized constraints. Some merit function is reduced along each search direction to ensure convergence from any starting point.

$$
\begin{cases}\text { Minimize } & f(X), X \in \mathfrak{R} \Rightarrow \\ & \min _{q}(d)=\frac{1}{2} d^{T} H_{i} d+\nabla f\left(X_{i}\right)^{T} d \\ \text { Subject to } & h(X)=0 \Rightarrow \nabla g\left(X_{i}\right)^{T} d+g\left(X_{i}\right) \leq 0 \\ & g(X)=0 \Rightarrow \nabla h\left(X_{i}\right)^{T} d+h\left(X_{i}\right)=0\end{cases}
$$

At each iterate $i$ a QP sub problem is used to generate a search direction $d$ towards the next iterate $X_{i+1}$

$$
X_{i+1}=X_{i}+\alpha_{i} d
$$

where the value of $\alpha_{i}$ is determinate in each iteration $i$ using one-dimensional minimization method.

\subsection{Multi Start Algorithm}

The multi start method for global optimization can overcome some of the limitations of the local Solving method. This method will automatically run the local method from a number of starting points and will display the best of several locally optimal solutions found, as the probable global optimal solution. The multi start algorithm attempts to find a global solution by starting a local solver from multiple starting points in the space of research S. This method generates uniformly distributed points in S, and starts local solver from each of these. This converges to a global solution.

\subsection{Proposed Algorithm: MSQP}

The MSQP Algorithm is a global optimization algorithm; this algorithm is the result of the combination of Multi start algorithm and the SQP method. It resumes the principal mechanisms of the SQP method to which are added other mechanisms destined to treat multi-modales problems. The solutions found by the SQP method during the execution of each iteration are improved, so that always we keeps the global solution and the local solution is ignored. Thus, at the end of the treatment, we obtain the global solution, that is the solution of the problem multimodal. In MSQP algorithm, the aspect of the global search of start Multi algorithm is used to maintain the diversity. In fact, we considering that MSQP algorithm will converge after a small number of iterations to a local solution if we find another local solution, we can consider that it is not useful to continue the search from this moment, and it is better to start a new search.

\subsection{Results}

In order to evaluate the performance of the method proposed, we compare the solutions obtained by the MSQP algorithm with the solutions reported in (pPSA: perturbed Particle Swarm Algorithm and pPSA best [15] for certain functions tests. Table 2 presents different solutions obtained by algorithms pPSA, pPSA best and proposed algorithm MSQP (see Table 2).

The computational results of MSQP algorithm and the algorithms PSO and TRIBES (that are cited in [16]), are summarized in tables for each example problem. The different results are obtained for a dimension $\mathrm{D}=10$ for every function test (see Table 3).

In this section, the different results are reported. The 
Table 2. The solutions obtained by pPSA, pPSA best and MSQP.

\begin{tabular}{ccccc}
\hline Functions & npart & pPSA & pPSA & MSQP \\
\hline \multirow{2}{*}{ Sphere } & 2 & $5.96 \mathrm{e}-6$ & $4.39 \mathrm{e}-6$ & $4.3216 \mathrm{e}-15$ \\
& 20 & $6.12 \mathrm{e}-6$ & $4.7 \mathrm{e}-6$ & $7.1525 \mathrm{e}-15$ \\
Quadratique & 2 & $1.47 \mathrm{e}+4$ & $8.06 \mathrm{e}-6$ & $7.3985 \mathrm{e}-17$ \\
& 20 & $3.56 \mathrm{e}-5$ & $9.04 \mathrm{e}-6$ & $3.5531 \mathrm{e}-8$ \\
Rastrigin & 2 & $1.78 \mathrm{e}+2$ & $8.76 \mathrm{e}+1$ & 0.9950 \\
& 20 & 85.9 & 54.7 & 7.9597 \\
Ackley & 2 & $1.83 \mathrm{e}+1$ & $1.64 \mathrm{e}+1$ & $5.6056 \mathrm{e}-7$ \\
& 20 & $7.59 \mathrm{e}-1$ & $1.59 \mathrm{e}-3$ & $1.6215 \mathrm{e}-5$ \\
\hline
\end{tabular}

Table 3. The solutions obtained by MSQP, TRIBES and PSO.

\begin{tabular}{lccc}
\hline Function & PSO & TRIBES & QPMS \\
\hline Rastrigin & $4.02(100000)$ & $8.5(100000)$ & $2.9849(100000)$ \\
Rosenbrock & $1.88(100000)$ & $0.06(1500000)$ & $0.7698(100000)$ \\
Ackley & $20.11(100000)$ & $20.32(100000)$ & $17.0918(100000)$ \\
\hline
\end{tabular}

proposed MSQP technique was tested on a number of benchmark multimodal mathematical functions and the performance compared with other Global Optimization approaches. The results finding by MSQP algorithm are the best ones for the most functions test.

\subsection{Structural Optimization under Reliability Constraints}

To illustrate the efficacy of the presented algorithm (see Figure 3) to solve the problems of structural design optimization under reliability constraint, we choose as example the truss constituted by six identical bars. For this problem, the objective function to minimize is the section A of each bars of the considered structure; the variables of conception are the dimensions of the section (the height and the width) (see Figure 4).

The geometric and material properties are:

- The initial section of every bar $A=0.0015 \mathrm{~m}^{2}$;

- The length $L=10 \mathrm{~m}$;

- The load $F=10 \mathrm{~N}$;

- The Young's modulus $E=2 \times 10^{8} \mathrm{~N} / \mathrm{m}^{2}$.

The minimization of the section is done under constraints on the design variables and under a reliability constraint (the probability of failure of the structure must not surpass a value limit). The formulation of the optimization problem for the section of each bar of truss is the following:

$$
\begin{aligned}
& \min _{w, t} f=w^{*} t \\
& \text { Subject to } \quad w \geq 1 \text { and } t \leq 5 \\
& 6.32 \leq w+t \leq 6.33 \\
& P_{f}=\frac{0.22}{w^{*} t} \leq 0.001
\end{aligned}
$$

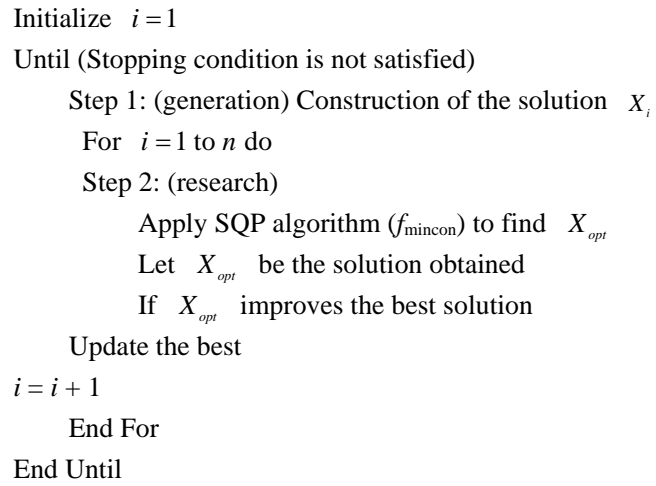

Figure 3. The basic steps of the MSQP algorithm.

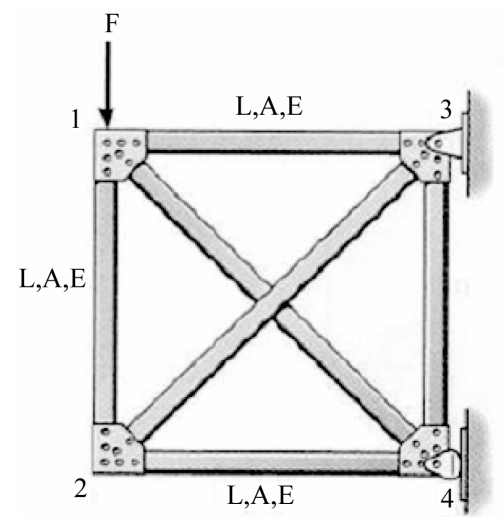

Figure 4. Truss constituted by 6 bars.

where $\mathrm{w}$ is the width and $t$ is the height.

To resolve this problem of structural optimization under reliability constraint, we used the algorithm MSQP developed in the preceding sections. Therefore, we obtained as results of this problem the following values: $w=$ $1.32 \mathrm{~cm}, t=5 \mathrm{~cm}$ and $A=6.6 \mathrm{e}-4 \mathrm{~m}^{2}$.

\section{Conclusion}

In this paper, an efficient, accurate, robust algorithm is proposed to solve the reliability problem with implicit response functions. The proposed algorithm integrates the treatment by the Finite Element Analysis method with FEA Software and the Probabilistic Transformation Method PTM (reliability program). In the proposed method, the Finite Element Analysis is used to approximate the structural response function. Once the implicit response function is found numerically, the Probabilistic Transformation Method PTM can easily be applied to solve the complicated structural reliability problem. The accuracy and efficiency of the proposed method is demonstrated through numerical examples of structures. A new global optimization algorithm MSQP is proposed. The new algorithm can be widely applied to a class of global optimization problems. The numerical results for some test functions show that the present algorithm has 
proved the robustness and high performance. The proposed algorithm MSQP is applied to solve structural problems under reliability constraints.

\section{REFERENCES}

[1] M. Lemaire, "Evaluation of Reliability Index Associated to Structural Mechanical Models," French Journal of Mechanic, Vol. 2, 1992, pp. 145-154.

[2] B. Sudreta and A. Der Kiureghian, "Comparison of Finite Element Reliability Methods,” Probabilistic Engineering Mechanics, Vol. 17, No. 4, 2002, pp. 337-348. doi:10.1016/S0266-8920(02)00031-0

[3] A. Der Kiureghian and J.-B. Ke, “The Stochastic Finite Element Method in Structural Reliability,” Probabilistic Engineering Mechanics, Vol. 3, No. 2, 1988, pp. 83-91. doi:10.1016/0266-8920(88)90019-7

[4] A. M. Hasofer and N. C. Lind, "Exact and Invariant Second-Moment Code Format," Journal of the Engineering Mechanics Division, Vol. 100, No. 1, 1974, pp. 111-121.

[5] H. O. Madsen, S. Kenk and N. C. Lind, "Methods of Structural Safety,” Prentice-Hall Inc., Upper Saddle River, 1986.

[6] R. Rackwitz, "Reliability Analysis: A Review and Some Perspectives," Structural Safety, Vol. 23, No. 4, 2001, pp. 365-395. doi:10.1016/S0167-4730(02)00009-7

[7] M. Lemaire, A. Mohamed and O. Flores-Macias, "The Use of Finite Element Codes for the Reliability of Structural Systems," 1997.

[8] A. Mohamed and M. Lemaire, "Linearzed Mechanical

\section{Notation}

PTM: Probabilistic Transformation Method.

FE: Finite Element.

PDF: Probability Density Function.

FEA: Finite Element Analysis.

FEACPTM: Finite Element Analysis Combined to Probabilistic Transformation Method.
Model to Evaluate Reliability off Shore Structures,” Structural Safety, Vol. 17, No. 3, 1995, pp. 167-193. doi:10.1016/0167-4730(95)00009-S

[9] S. Kadry, "A Proposed Technique to Evaluate the Stochastic Mechanical Response Based on Transformation with Finite Element Method," International Journal of Applied Mathematics and Mechanics, Vol. 2, No. 2, 2006, pp. 94-108.

[10] G. Muscolino, G. Ricciardi and N. Impollonia, "Improved Dynamic Analysis of Structures with Mechanical Uncertainties under Deterministic Input," Structural Safety, Vol. 15, No. 2, 2000, pp. 199-212.

[11] European Committee for Standardization, "Eurocode 3: Design of Steel Structures,” Eyrolles, Paris, 1992.

[12] P. Siarry, J. Dréo, A. Pétrowski and E. Taillard, "Metaheuristics for Hard Optimization,” Eyrolles, Paris, 2003.

[13] R. Fletcher, "Practical Methods of Optimization,” 2nd Edition, John Wiley and Sons, Hoboken, 2000.

[14] Z. Xinchao, "A Perturbed Particle Swarm Algorithm for Numerical Optimization,” Applied Soft Computing, Vol. 10, No. 1, 2010, pp. 119-124. doi:10.1016/j.asoc.2009.06.010

[15] Y. Cooren, "Development of an Adaptive Algorithm of Particulate Swarm Optimization. Applications in Medical Engineering and Electronics,” Ph.D. Thesis, 12 Val de Marne University, Paris, 2008.

[16] A. Mohamed and M. Lemaire, "Linearized Mechanical Model to Evaluate Reliability of Offshore Structures," Structural Safety, Vol. 17, 1995, pp. 167-193.

FORM: First Order Reliability Method.

SORM: Second Order Reliability Method.

$G($.$) : Limit State function.$

$P_{f}$ : Probability of failure.

SQP: Sequential Quadratic Programming.

MSQP: Multi start algorithm combined to Sequential Quadratic Programming method. 